\title{
A FUNCTIONAL ANALYSIS PROOF OF THE EXISTENCE OF HAAR MEASURE ON LOCALLY COMPACT ABELIAN GROUPS
}

\author{
ALEXANDER J. IZZO
}

(Communicated by Andrew M. Bruckner)

\begin{abstract}
A simple proof of the existence of Haar measure on locally compact abelian groups is given. The proof uses the Markov-Kakutani fixed-point theorem.
\end{abstract}

It is very well known that every locally compact group has a Haar measure and that the Haar measure is unique up to a positive multiplicative constant. Several different proofs have been given, all of them somewhat difficult. (See [N] for two proofs as well as references to others). In most of these proofs, the existence and uniqueness of Haar measure are established separately. For compact groups, a simple proof of the existence and uniqueness of Haar measure was given by von Neumann [vN], and his proof can be made even simpler by using the Kakutani fixed-point theorem (see [R2]). For locally compact abelian groups, uniqueness of Haar measure is easily established (see [R1, p. 2]). The purpose of this short note is to present a simple proof of the existence of Haar measure for these groups. The proof will make use of the Markov-Kakutani fixed-point theorem, which we recall below. It is known that this fixed-point theorem can be used to prove that every locally compact abelian group has an invariant mean (see [P, p. 113]). For compact groups an invariant mean and a Haar measure are the same thing, but for noncompact groups this is obviously not the case.

Theorem (Markov-Kakutani). Let $K$ be a nonempty compact convex subset of a (Hausdorff) topological vector space. Let $\mathscr{F}$ be a commuting family of continuous affine mappings of $K$ into itself. Then there exists a point $p \in K$ such that $T p=p$ for all $T \in \mathscr{F}$.

A proof can be found in [C, pp. 155-156]. (There the theorem is stated only for locally convex spaces, but local convexity is not needed in the proof.)

We will also need two lemmas.

Received by the editors November 28, 1990.

1991 Mathematics Subject Classification. Frimary 28C10, 43A05, 22B05; Secondary 46N99.

Key words and phrases. Haar measure, locally compact abelian groups, Markov-Kakutani fixedpoint theorem.

This research was partially supported by NSF grant DMS 90-02904.

(C) 1992 American Mathematical Society $0002-9939 / 92 \$ 1.00+\$ .25$ per page 
Lemma 1. Suppose $G$ is a topological group and $N$ is a neighborhood of the identity in $G$ that is symmetric (i.e., $N^{-1}=N$ ). Then there exists a subset $S$ of $G$ such that for each $g$ in $G$ the set $g N \cdot N$ contains at least one element of $S$ and the set $g N$ contains at most one element of $S$.

Proof. Let $\mathscr{G}$ be the collection of all subsets $T$ of $G$ such that

$$
p^{-1} q \notin N \cdot N \text { for all } p, q \in T \text {. }
$$

By applying Zorn's lemma, we see that $\mathscr{G}$ has a maximal element $S$. Now if $g \in G$, then there is some $s$ in $S$ such that $g^{-1} s \in N \cdot N$, for otherwise the set $S \cup\{g\}$ would be a member of $\mathscr{G}$ strictly containing $S$. Moreover, if there were two distinct points $s_{1}, s_{2}$ in $S$ such that both $g^{-1} s_{1}$ and $g^{-1} s_{2}$ were in $N$, then we would have $s_{1}^{-1} s_{2}=s_{1}^{-1} g g^{-1} s_{2} \in N^{-1} \cdot N=N \cdot N$, a contradiction. Thus, there is at most one $s$ in $S$ such that $g^{-1} s \in N$.

Lemma 2. Let $X$ be a vector space, and let $X^{*}$ denote the space of all linear functionals on $X$ with the weak*-topology (i.e., the weak topology induced by $X)$. If $K$ is a closed subset of $X^{*}$ such that for each $x \in X$ the set $\{\Lambda x: \Lambda \in K\}$ is bounded, then $K$ is compact.

The proof of this lemma is very similar to the proof of the Banach-Alaoglu theorem and is essentially contained in [DS, pp. 423-424]. A more succinct statement of the conclusion is that every closed bounded set in $X^{*}$ is compact.

Proof of the existence of Haar measure on locally compact abelian groups. Let $G$ be a locally compact abelian group. Let $C_{c}(G)$ denote the space of compactly supported continuous functions on $G$, and let $C_{c}(G)^{*}$ denote the space of all linear functionals on $C_{c}(G)$ with the weak*-topology (i.e., the weak topology induced by $\left.C_{c}(G)\right)$. If $f \in C_{c}(G)$ and $a \in G$, then $f_{a}$ (the translate of $f$ by $a)$ is defined by $f_{a}(x)=f(a+x)$. For each $a$ in $G$, define $T_{a}: C_{c}(G)^{*} \rightarrow C_{c}(G)^{*}$ by the equation

$$
\left(T_{a} \Lambda\right)(f)=\Lambda\left(f_{a}\right) \quad\left(\Lambda \in C_{c}(G)^{*}, f \in C_{c}(G)\right) .
$$

Then each $T_{a}$ is a continuous linear operator. To establish the existence of Haar measure on $G$ we must simply show that there is a nonzero positive linear functional on $C_{c}(G)$ that is fixed by every $T_{a}$.

Fix a symmetric neighborhood $N$ of the identity in $G$ with compact closure. Let $K$ be the set of positive linear functionals $\Lambda$ on $C_{c}(G)$ that satisfy the following two conditions:

(i) $\Lambda(f) \leq 1$ whenever $f$ is a nonnegative function in $C_{c}(G)$ that is bounded above by 1 and whose support is contained in $a+N$ for some $a \in G$, and

(ii) $\Lambda(f) \geq 1$ whenever $f$ is a nonnegative function in $C_{c}(G)$ that is equal to 1 on $a+N+N$ for some $a \in G$.

Then $K$ is clearly closed and convex in $C_{c}(G)^{*}$. Moreover, by a partition of unity argument every nonnegative function in $C_{c}(G)$ can be written as a finite sum of nonnegative continuous functions each of which has support in $a+N$ for some $a \in G$. It follows that condition (i) in the definition of $K$ implies that for each function $f$ in $C_{c}(G)$, the set $\{\Lambda(f): \Lambda \in K\}$ is bounded. Therefore by Lemma $2, K$ is compact. 
Let $S$ be as in Lemma 1, and note that the functional that consists of a point mass at each point of $S$ (i.e., the functional $f \mapsto \sum_{s \in S} f(s)$ ) is in $K$. Thus $K$ is nonempty.

It is clear from the definition of $K$ that each of the operators $T_{a}$ maps $K$ into itself. Hence, since the operators $T_{a}(a \in G)$ form a commuting family, the Markov-Kakutani fixed-point theorem shows that they have a common fixedpoint in $K$. Since all the elements of $K$ are nonzero positive linear functionals on $C_{c}(G)$, the proof is complete.

\section{REFERENCES}

[C] J. B. Conway, A course in functional analysis, Springer-Verlag, New York, 1985.

[DS] N. Dunford and J. Schwartz, Linear operators, Part I, Interscience Publ., New York, 1958.

[N] L. Nachbin, The Haar integral, D. Van Nostrand Co., Princeton, NJ, 1965.

[vN] J. von Neumann, Zum Haarschen mass in topologischen gruppen, Comp. Math. 1 (1934), 106-114.

[P] J. P. Pier, Amenable locally compact groups, Interscience Publ., New York, 1984.

[R1] W. Rudin, Fourier analysis on groups, Interscience Publ., New York, 1962.

[R2] _ Functional analysis, McGraw-Hill, New York, 1973.

Department of Mathematics, University of Michigan, Ann Arbor, Michigan 48109

E-mail address: izzo@math.lsa.umich.edu 